PDES, SUBMANIFOLDS AND

AFFINE DIFFERENTIAL GEOMETRY

BANACH CENTER PUBLICATIONS, VOLUME 57

INSTITUTE OF MATHEMATICS

POLISH ACADEMY OF SCIENCES

WARSZAWA 2002

\title{
AFFINOR STRUCTURES IN THE OSCILLATION THEORY
}

\author{
BORIS N. SHAPUKOV \\ Department of Geometry, University of Kazan \\ 420008 Kazan, Russia \\ E-mail: Boris.Shapukov@ksu.ru
}

\begin{abstract}
In this paper we consider the system of Hamiltonian differential equations, which determines small oscillations of a dynamical system with $n$ parameters. We demonstrate that this system determines an affinor structure $J$ on the phase space $T \mathbf{R}^{n}$. If $J^{2}=\omega I$, where $\omega= \pm 1,0$, the phase space can be considered as the biplanar space of elliptic, hyperbolic or parabolic type. In the Euclidean case $\left(\mathbf{R}^{n}=E^{n}\right)$ we obtain the Hopf bundle and its analogs. The bases of these bundles are, respectively, the projective $(n-1)$-dimensional spaces over algebras of complex, double and dual numbers.
\end{abstract}

1. Introduction. Geometry and topology have various connections with analytical mechanics. The Newtonian mechanics essentially uses the Euclidean structure of space, and the relativistic mechanics used from the outset the Minkowski geometry that had been already developed. On the other hand, the Riemannian geometry was initiated not only by the relativity theory, but by applications to the mechanics of conservative systems, as well. The geometrical interpretation of Euler-Lagrange equations and the Calculus of Variations gave rise to the Finsler geometry and its generalizations. It should be noted that the determining equations generate geometrical structures on associated spaces. In the last three decades new essential results were achieved in the field of Hamiltonian mechanics with the use of algebraic, geometrical, and topological methods. In this connection we should mention results due to V. Arnold [1], J. Marsden and A. Weinstein [2], A. Fomenko [3] et al., on integration of Hamiltonian equations, admitting symmetry groups.

We demonstrate the way geometry appears in oscillation theory. In what follows we shall restrict our consideration to small oscillations. Our basic assumptions are those given in [4].

2000 Mathematics Subject Classification: Primary 53C15; Secondary 53C80.

Key words and phrases: oscillation, affinor structure, biplanar space, Hopf bundle.

Research supported by RFFI grant 00-01-00308.

The paper is in final form and no version of it will be published elsewhere. 
1) The system under consideration depends on $n$ parameters, and is conservative. This means that we have a Lagrangian manifold $M$, and, with respect to Lagrangian coordinates $x=\left(x^{i}\right)$, the kinetic energy $T$ and the potential energy $V$ are written as

$$
T=\frac{1}{2} a_{i j}(x) \dot{x}^{i} \dot{x}^{j}>0, \quad V=V(x) .
$$

Recall that $T$ endows $M$ with a Riemannian metric $d s^{2}=a_{i j}(x) d x^{i} d x^{j}$. A point $x_{0} \in M$ is called an equilibrium position if $\left.\operatorname{grad} V\right|_{x_{0}}=0$. Certainly, we can suppose that this point has zero coordinates, i. e. $x_{0}^{i}=0$.

2) At the initial time the system is in a neighborhood of $x_{0}$, and the initial velocity is small. Then, up to second order, we get the pair of quadratic forms with constant coefficients

$$
T=\frac{1}{2} a_{i j} \dot{x}^{i} \dot{x}^{j}=\frac{1}{2}(A \dot{x}, \dot{x}), \quad V=\frac{1}{2} b_{i j} x^{i} x^{j}=\frac{1}{2}(B x, x) .
$$

From the geometrical point of view, this means that in a small neighbourhood of $x_{0}$ the Riemannian manifold $M$ can be replaced with the tangent plane endowed with the Euclidean metric. In $T_{x_{0}} M$ we shall use the induced Cartesian coordinates $\left(x^{i}\right)$. Thus locally we obtain a linearization of the problem. Then we also get a linearization of the Lagrange equations

$$
\frac{d}{d t} \frac{\partial T}{\partial \dot{x}^{i}}-\frac{\partial T}{\partial x^{i}}=-\frac{\partial V}{\partial x^{i}},
$$

the following linear system of ordinary differential equations (ODE)

$$
\ddot{x}=C x, \quad C=-A^{-1} B .
$$

Note that the vector field $C x$ is potential if and only if the operator $C$ is symmetric.

The system (3) is equivalent to the system of $2 n$ equations

$$
\dot{x}=y, \quad \dot{y}=C x .
$$

or, if we set $z=(x, y)$, to the system

$$
\dot{z}=J z, \quad J=\left(\begin{array}{cc}
0 & I_{n} \\
C_{n} & 0
\end{array}\right),
$$

defined on the phase space. Note that this bundle with the canonical projection $p$ : $T E^{n} \rightarrow E^{n}$ is trivial and its total space is isomorphic to the product $E^{n} \times E^{n}=E^{2 n}$ with the metric $z^{2}=x^{2}+y^{2}$.

Each solution of (4) is a phase curve, an integral curve of the vector field $v=(y, C x)$, i.e. an orbit of one-parameter group of transformations $z(t)=\exp (t v) z$. Since the system is conservative, the curve $z(t)$ lies on a level surface of constant total energy $E=T+V$. For the initial data $z(0)=z$, the solution is $z(t)=e^{J t} z$. Then the curve $x(t)=p z(t)$ is a solution of the equations (3).

We shall use some algebraic notions (for details see [5]). Each two-dimensional associative commutative algebra with unit has the form $\mathbf{A}=\left\{a=a_{1}+\kappa a_{2} \mid \kappa^{2}=\omega= \pm 1,0\right\}$, where $a_{1}, a_{2} \in \mathbf{R}$, and $1, \kappa$ are basic elements of $\mathbf{A}$. For $\omega=-1$ we get the algebra $\mathbf{C}=\mathbf{R}(i)$ of complex numbers, for $\omega=1$ the algebra $\mathbf{R}(e)$ of double numbers (or, of paracomplex numbers), and for $\omega=0$ the algebra $\mathbf{R}(\varepsilon)$ of dual numbers. 
Let us consider an $\mathbf{R}$-linear representation of $\mathbf{A}$ in the algebra of endomorphisms of $\mathbf{R}^{2 n}$ such that $1 \rightarrow I=I d, \kappa \rightarrow J$. This representation is uniquely determined by the affinor (linear operator) $J$ such that $J^{2}=\omega I$. The affinor structure $J$ is said to be of elliptic type if $\omega=-1$, of hyperbolic type if $\omega=1$, and of parabolic type if $\omega=0$. Let $G \subset G L(2 n, \mathbf{R})$ be the subgroup of linear automorphisms of $\mathbf{R}^{2 n}$ which commute with $J$. Then the Klein space $\left(\mathbf{R}^{2 n}, G\right)$ is called a biplanar space of the corresponding type. A canonical basis $\left\{e_{1}, \ldots, e_{n}, J e_{1}, \ldots, J e_{n}\right\}$ such that the affinor $J$ has the matrix

$$
J=\left(\begin{array}{cc}
0 & I_{n} \\
\omega I_{n} & 0
\end{array}\right),
$$

where $I_{n}$ is the identity $n \times n$-matrix, is defined up to transformations of $G$. With respect to this basis, each vector $z \in \mathbf{R}^{2 n}$ can be written as $z=(x, y), x, y \in \mathbf{R}^{n}$.

A biplanar space can be considered as a module $L^{n}(\mathbf{A})$ over $\mathbf{A}$. The multiplication is given as follows:

$$
\left(a_{1}+\kappa a_{2}\right) z=a_{1} z+a_{2} J z, \quad \forall z \in \mathbf{R}^{2 n} .
$$

Conversely, for a finitely generated free $\mathbf{A}$-module $L^{n}(\mathbf{A})$, one can consider the vector space $L^{n}(\mathbf{A})$ over $\mathbf{R}$ as a biplanar space $\mathbf{R}^{2 n}$ with affinor $J z=\kappa z$. Thus we can write $z=x+\kappa y$ rather than $z=(x, y)$.

2. Simple examples. The following two examples are given in [1] (pp. 23-24).

a) Let $n=1$. The equation $\ddot{x}=-x$ describes an oscillation in a neighborhood of the equilibrium $0 \in E^{1}$. In the phase space this equation generates the system

$$
\dot{x}=y, \dot{y}=-x \text {. }
$$

In this case the affinor structure $J$ has the form $J=\left(\begin{array}{cc}0 & 1 \\ -1 & 0\end{array}\right), J^{2}=-I$, and is an operator of complex structure in $T E^{1}$. We have $T=\frac{1}{2} \dot{x}^{2}, V=\frac{1}{2} x^{2}, E=\frac{1}{2}\left(x^{2}+y^{2}\right)$. In terms of complex variables, if we set $z=x+i y,(6)$ is written as $\dot{z}=-i z$. For the initial condition $z(0)=z$ the solution is the phase curve $z(t)=e^{-i t} z$, or, in terms of real variables,

$$
x(t)=x \cos t+y \sin t, \quad y(t)=-x \sin t+y \cos t .
$$

These are circles in $E^{2}$, which are orbits of the group $S O(2)$. Note that in this case the first integral gives the complete solution of the problem: $2 E=x^{2}+y^{2}=R^{2}$.

b) Let $n=2$. The equations $\ddot{x}=-x, x=\left(x^{1}, x^{2}\right)$ describe the oscillation of a spherical pendulum in $E^{2}$. In the phase space they give the system

$$
\dot{x}^{1}=y^{1}, \dot{x}^{2}=y^{2}, \dot{y}^{1}=-x^{1}, \dot{y}^{2}=-x^{2} .
$$

In this case $z=\left(x^{1}, x^{2}, y^{1}, y^{2}\right)$, and the affinor structure $J=\left(\begin{array}{cc}0 & I_{2} \\ -I_{2} & 0\end{array}\right), J^{2}=-I$, is a complex structure in $T E^{2}$. We have

$$
T=\frac{1}{2}\left(\left(\dot{x}^{1}\right)^{2}+\left(\dot{x}^{2}\right)^{2}\right), \quad V=\frac{1}{2}\left(\left(x^{1}\right)^{2}+\left(x^{2}\right)^{2}\right) .
$$

Here it is useful to use the complex variables. If we set $z^{k}=x^{k}+i y^{k}$, then, according to (7), we have $\dot{z}^{k}=-i z^{k}$. From this we obtain that $z^{k}(t)=e^{-i t} z^{k}$. Each curve lies on 
a sphere $S^{3}(R) \subset T E^{2}$

$$
2 E=\left(x^{1}\right)^{2}+\left(x^{2}\right)^{2}+\left(y^{1}\right)^{2}+\left(y^{2}\right)^{2}=R^{2},
$$

and is an orbit of a subgroup of rotations $S O(2) \subset S O(3)$. Finally, for each $R$ we have the principal Hopf bundle $\pi_{R}: S^{3}(R) \rightarrow S^{2}$ with projection $\pi_{R}:\left(z^{1}, z^{2}\right) \rightarrow\left(z^{1}: z^{2}\right)$. Note that $S^{2}$ is diffeomorphic to the complex projective line $\mathbf{C} P^{1}$. The other projection $p: T E^{2} \rightarrow E^{2}$ maps each phase curve to an integral curve of the original equation. The integral curves are ellipses. For the initial conditions $x=\left(c^{1}, c^{2}\right), y=\left(c^{3}, c^{4}\right)$, they have the equations

$$
\left(\left(c^{2}\right)^{2}+\left(c^{4}\right)^{2}\right)\left(x^{1}\right)^{2}+\left(\left(c^{1}\right)^{2}+\left(c^{3}\right)^{2}\right)\left(x^{2}\right)^{2}-2\left(c^{1} c^{2}+c^{3} c^{4}\right) x^{1} x^{2}+\left(c^{2} c^{3}-c^{1} c^{4}\right)^{2}=0 .
$$

3. Biplanar structures and analogs of the Hopf bundle. Now, regardless of the oscillation theory, we shall consider the second order linear system of ODE

$$
\ddot{x}=C x, \quad x=\left(x^{1}, x^{2}, \ldots x^{n}\right) \in \mathbf{R}^{n},
$$

where $C$ is a real $n \times n$-matrix. Further, let us consider the phase space of this system, i.e. $T \mathbf{R}^{n}$. This is a trivial bundle over $\mathbf{R}^{\mathbf{n}}$, and $T \mathbf{R}^{n} \cong \mathbf{R}^{n} \times \mathbf{R}^{n}$. We set $y=\dot{x}$, and write this system in the form (4).

Now we shall investigate special cases of affinor structures in $T \mathbf{R}^{n}$, which are of geometric interest. Suppose $J$ is such that $J^{2}=\omega I$, where $\omega= \pm 1,0$. Then the following three cases are possible:

$$
\text { a) } \left.\left.C=-I_{n}, b\right) C=I_{n}, c\right) C=0 .
$$

a) We obtain the system

$$
\dot{x}=y, \dot{y}=-x, J=\left(\begin{array}{cc}
0 & I_{n} \\
-I_{n} & 0
\end{array}\right) .
$$

This affinor defines a complex structure in the phase space $T \mathbf{R}^{n}$, and we obtain the biplanar space $B^{2 n}$ of elliptic type. Note that the pair $\{I, J\}$ gives a representation of the algebra $\mathbf{C}=\mathbf{R}(i)$. This space contains a pair of complex conjugate $n$-eigenplanes (the absolute planes) $x+i y=0$ and $x-i y=0$, which correspond to $n$-multiple eigenvalues $\lambda= \pm i$ of $J$. Therefore, it is convenient to consider the complex vector space $L^{n}(i)$ with complex coordinates $z^{k}=x^{k}+i y^{k}$. Then complex straight lines $L(z)=\{z, i z\}, z \neq 0$, stratify the space $L_{0}^{n}(i)=L^{n}(i) \backslash\{0\}$, and give fibres of a nontrivial principal bundle $\pi: L_{0}^{n}(i) \rightarrow P^{n-1}(i)$ over the complex projective space with projection $\pi(z)=\left(z^{1}: z^{2}\right.$ : $\left.\ldots: z^{n}\right)$. The action of the structure group $\mathbf{C}_{0}=\mathbf{C} \backslash\{0\}$ is given by $z^{\prime}=a z$.

Then (9) takes the form $\dot{z}=-i z$, and the solutions are $z(t)=e^{-i t} z$. In terms of real geometry, we have a principal bundle $\pi: B_{0}^{2 n} \rightarrow P^{n-1}(i)$ whose fibres form a $2(n-1)$ parametric family of 2-dimensional planes $L^{2}(z)=\{z, J z\}$ (the absolute congruence) with the parametric equations $u=\lambda x+\mu y, v=-\mu x+\lambda y$. The phase curves have the equations

$$
x(t)=x \cos t+y \sin t, \quad y(t)=-x \sin t+y \cos t,
$$

and are orbits of a one-parameter group $S O(2)$. These curves lie on the 2-planes mentioned above. 
Now we take a Euclidean space $\mathbf{R}^{n}=E^{n}$ with metric $d s^{2}=2 T d t^{2}$ and the orthonormal coordinates $\left(x^{k}\right)$. As the operator $C$ is symmetric, there exists a potential $V=\frac{1}{2} \sum_{k=1}^{n}\left(x^{k}\right)^{2}$, and the system (9) has the first integral $E=T+V$. Each phase curve lies on a level hypersphere $S^{2 n-1}(R) \subset B_{0}^{2 n}$ given by the equations

$$
2 E=\sum_{k=1}^{n}\left(\left(x^{k}\right)^{2}+\left(y^{k}\right)^{2}\right)=R^{2} .
$$

Therefore, each phase curve is a section $L^{2}(z) \cap S^{2 n-1}(R)$, a great circle of hypersphere. Finally, for each radius $R$, we get the Hopf bundle $\pi_{R}: S^{2 n-1}(R) \rightarrow P^{n-1}(i)$.

b) We have the system

$$
\dot{x}=y, \dot{y}=x, J=\left(\begin{array}{cc}
0 & I_{n} \\
I_{n} & 0
\end{array}\right) .
$$

This case is analogous to the previous one. Since $J^{2}=I$, this affinor defines a double structure in the phase space $T \mathbf{R}^{n}$, and we get the biplanar space $B^{2 n}$ of hyperbolic type. The pair $\{I, J\}$ gives a representation of the algebra $\mathbf{R}(e)\left(e^{2}=1\right)$ of double numbers. $B^{2 n}$ contains a pair of real $n$-eigenplanes (the absolute planes) $x+y=0$ and $x-y=$ 0 , which correspond to the $n$-multiple eigenvalues $\lambda= \pm 1$ of $J$. We consider the free $\mathbf{R}(e)$-module $L^{n}(e)$ with coordinates $z^{k}=x^{k}+e y^{k}$. Then the free $\mathbf{R}(e)$-modules of rank 1 (straight lines) $L(z)=\{z, e z\},|z|^{2}=z \bar{z}=x^{2}-y^{2} \neq 0$ form a congruence in $L_{0}^{n}(i)=L^{n}(i) \backslash\{|z|=0\}$ and define a nontrivial principal bundle $\pi: L_{0}^{n}(e) \rightarrow P^{n-1}(e)$. The base is a projective space over the algebra of double numbers except the points corresponding to the isotropic straight lines which lie on the cone $|z|^{2}=x^{2}-y^{2}=0$. The structure group $\mathbf{R}_{0}(e)=\left\{a:|a|^{2} \neq 0\right\}$ is the Lie group of invertible elements of $\mathbf{R}(e)$, and the action of $\mathbf{R}_{0}(e)$ is $z^{\prime}=a z$.

In the double space the equations (10) take the form $\dot{z}=e z$, and their solutions are $z(t)=e^{e t} z$.

In terms of real geometry, we have a principal bundle $\pi: B_{0}^{2 n} \rightarrow P^{n-1}(e)$ whose fibres are the 2-planes $L^{2}(z)=\{z, J z\}$ with parametric equations $u=\lambda x+\mu y, v=\mu x+\lambda y$, $x^{2}-y^{2} \neq 0$. These fibres form a $2(n-1)$-parametric family (the absolute congruence). The solutions give the phase curves

$$
x(t)=x \cosh t+y \sinh t, \quad y(t)=x \sinh t+y \cosh t .
$$

These curves are orbits of the 1-parametric group $S O(1,1)$, and they lie in the 2-planes mentioned above.

Now suppose that the space is Euclidean. As operator $C$ is symmetric, there exists the potential $V=-\frac{1}{2} \sum_{k=1}^{n}\left(x^{k}\right)^{2}$, and the system (10) has the first integral $E=T+V$. The phase curves lie on the level surfaces which constitute a one-parameter family of hyperboloids $S_{n}^{2 n-1}(R)$ given by the equations

$$
2 E=\sum_{k=1}^{n}\left(-\left(x^{k}\right)^{2}+\left(y^{k}\right)^{2}\right)= \pm R^{2} .
$$

Therefore, the phase curves are diametrical sections $L^{2}(z) \cap S_{n}^{2 n-1}(R)$. These hyperboloids can be also considered as hyperspheres of real radius or pure imaginary radius in the 
pseudo-Euclidean space $E_{n}^{2 n}$ of type $(-n, n)$. Therefore we have a hyperbolic analog of the Hopf bundle $\pi_{R}: S_{n}^{2 n-1}(R) \rightarrow P^{n-1}(e)$.

c) We have the system

$$
\dot{x}=y, \dot{y}=0, J=\left(\begin{array}{cc}
0 & I_{n} \\
0 & 0
\end{array}\right) .
$$

This case is singular. Since $J^{2}=0$, this affinor defines a dual structure in the phase space $T \mathbf{R}^{n}$, and the pair $\{I, J\}$ gives a representation of the algebra $\mathbf{R}(\varepsilon),\left(\varepsilon^{2}=0\right)$ of a dual numbers. We obtain the biplanar space $B^{2 n}$ of parabolic type. This space contains the real $n$-eigenplane $y=0$ (the absolute plane) corresponding to the $n$-multiple eigenvalue $\lambda=0$ of $J$. We consider the free $\mathbf{R}(\varepsilon)$-module $L^{n}(\varepsilon)$ with coordinates $z^{k}=y^{k}+\varepsilon x^{k}, \varepsilon^{2}=0$. Then pairs $\{z, \varepsilon z\},|z|^{2}=y^{2} \neq 0$ define a congruence of dual straight lines of parabolic type in $L_{0}^{n}(\varepsilon)$. These lines are orbits of the Lie group $\mathbf{R}_{0}(\varepsilon)=\left\{a \in \mathbf{R}(\varepsilon):|a|^{2} \neq 0\right\}$ of invertible elements of the algebra $\mathbf{R}(\varepsilon)$. The action of this Lie group is $z^{\prime}=a z$. Note, that these straight lines are the fibres of a nontrivial principal bundle $\pi: L_{0}^{n}(\varepsilon) \rightarrow P_{0}^{n-1}(\varepsilon)$. The base is a dual projective space over the $\mathbf{R}(\varepsilon)$ except the points corresponding to isotropic straight lines, i. e. the the straight lines in the the absolute $n$-plane $|z|^{2}=y^{2}=0$.

In the dual space the equations (11) take the form $\dot{z}=\varepsilon z$, and, for the initial condition $z(0)=z$, we obtain $z(t)=e^{\varepsilon t} z$. Since $e^{\varepsilon t}=1+\varepsilon t$, we have that each straight line has the equation $z(t)=(1+\varepsilon t) z$.

In terms of real geometry, we have a principal bundle $\pi: B_{0}^{2 n} \rightarrow P_{0}^{n-1}(\varepsilon)$ whose fibres constitute the absolute congruence of 2-planes $L^{2}(z)$ with parametric equations $u=\lambda y, v=\lambda x+\mu y$. And the solutions give the straight lines $x(t)=x+t y, y(t)=y$. These are orbits of the one-parameter group $\mathbf{R}$ which lie in the absolute 2-planes. If the space $\mathbf{R}^{n}$ is Euclidean, these straight lines lie in the level surfaces

$$
Q: y^{2}=R^{2}, R \neq 0 \text {. }
$$

These surfaces form a one-parameter family of elliptic cylinders. Therefore, in this case each phase curve is the section $L^{2}(z) \cap Q$, i.e. the phase curves form a $2(n-1)$-parameter family of rectilinear generators of these cylinders. For any $R$ we again have a principal bundle $\pi_{R}: Q \rightarrow P_{0}^{n-1}(\varepsilon)$. Cylinders $Q$ can be also considered as hyperspheres in the semi-Euclidean space. Therefore we have a parabolic analog of the Hopf bundle.

Thus, we arrive at the following results.

THEOREM 1. The phase space of oscillator (8), where $C= \pm I_{n}, 0$, is a biplanar space of elliptic, hyperbolic, or parabolic type.

THEOREM 2. The phase curves of oscillator (8) fibre the level surfaces of total energy. Each level surface is the total space of a principal fibre bundle whose base is a projective $(n-1)$-dimensional space over the algebra of complex, double, or dual numbers.

4. Unsolved problems. First of all, it is important to investigate other affine structures. For example, since

$$
J^{2 k}=\left(\begin{array}{cc}
C^{k} & 0 \\
0 & C^{k}
\end{array}\right), \quad J^{2 k+1}=\left(\begin{array}{lc}
0 & (-1)^{k+1} C^{k} \\
C^{k+1} & 0
\end{array}\right)
$$


for affinors such that $C^{k}=\omega I_{n}, k<n$, the phase space is possibly fibred by $k$-planes containing phase curves, and we get other analogs of Hopf bundles.

It is interesting to study small oscillations in the pseudo-Euclidean space. The results are of importance for Special Relativity. In this case the kinetic energy of the system is an indefinite quadratic form. Hence the affinor structure $J$ which arises in the phase space in general has not only real eigenvalues but complex ones, as well. This situation also occurs in the general theory of linear differential equations.

The more interesting problem is to apply geometrical methods to non-linear oscillations. In the framework of conservative systems the kinetic and potential energy given by (1) determine a Riemannian space with metric $d s^{2}=2(E-V) a_{i j} d x^{i} d x^{j}$, where the system trajectories are geodesic lines. Then Geometry gives a possibility to connect non-linear effects with Riemannian curvature.

\section{References}

[1] V. I. Arnold, Mathematical Methods in Classical Mechanics, Nauka, Moscow, 1974 (in Russian).

[2] J. Marsden and A. Weinstein, Reduction on symplectic of manifolds with symmetry, Rep. Math. Phys. 5 (1974), 121-130.

[3] V. V. Trofimov and A. T. Fomenko, Algebra and Geometry of Integrable Hamiltonian Differential Equations, Faktorial - Prosperus, 1995 (in Russian).

[4] F. R. Gantmaher, Lectures on Analytical Mechanics, Nauka, Moscow, 1964 (in Russian).

[5] V. V. Vishnevskii, A. P. Shirokov and V. V. Shurygin, Spaces over Algebras, Kazan, 1985 (in Russian). 